\title{
Net Polarization of a Molecular Beam by Strong Electrostatic or Radiative Fields
}

\author{
Bretislav Friedrich \\ Fritz-Haber-Institut der Max-Planck-Gesellschaft \\ Faradayweg 4-6, D-14195 Berlin, Germany
}

\begin{abstract}
We present a simple analytic approximation for evaluating the ensemble-averaged orientation or alignment of a beam of molecules subjected to a strong static or radiative field. This approximation is based on the eigenproperties which polar or polarizable molecules exhibit in the strong-field, harmoniclibrator limit, and on the Boltzmann statistics of the free rotor states which adiabatically correlate with the harmonic librator states. For either the permanent or induced dipole case, the resultant formula involves just two dimensionless parameters which characterize the strength of the molecule-field interaction and the rotational temperature. The net polarization of a molecular beam thus obtained is shown to be in an excellent agreement with the exact values computed numerically from first principles. The validity range of the approximation includes the large-interaction, high temperature regions of the parameter space where first-principle calculations are onerous.
\end{abstract}

PACS: $32.60 .+\mathrm{i}$ Zeeman and Stark effects, 39.10.+j Atomic and molecular beam sources and techniques 


\section{Introduction}

When subject to an external field, an ensemble of molecules becomes polarized. The degree to which this polarization takes place depends on the interaction of the individual molecules with the field, as well as on the distribution of the molecules over the molecular states available.

The problem of the polarization of an equilibrium ensemble of magnetic or electric dipoles interacting with a static electric or magnetic field was first treated by Langevin [1] and Debye [2], and subsequently by Van Vleck [3]. Their work, which covered the high-temperature, low-field limit case, was extended by Friedrich and Herschbach [4] to encompass essentially all field strengths and temperatures. In addition, Friedrich and Herschbach tackled the case of the induced-dipole interaction, ignored in previous treatments. In all this work, the populations of the molecular states in the field were derived from Boltzmann factors based on eigenenergies which the molecules attain in the field. Thus the approximations reaped from these treatments pertain to ensembles of molecules that reach equilibrium within the field.

In this paper we deal likewise with the polarization of an ensemble of polar or polarizable molecules interacting with an electrostatic or radiative field; however, here we consider the instance when the populations of the molecular states in the field are given by the populations of the field-free rotor states. This situation corresponds to the case of beam molecules which enter adiabatically into an electric or radiative field from a field-free region. Needless to say, such a situation is encountered in many current experiments.

The analytic approximation for the net polarization that we here develop is based on the eigenproperties of molecules in the strong-field, harmonic-librator limit, and on the Boltzmann statistics of the free rotor states which adiabatically correlate with the harmonic librator states.

The approximation gives rise to simple, yet accurate formulae for the net orientation and alignment of a molecular beam. Their applicability range is assessed by a comparison with exact numerical calculations.

\section{Eigenproperties of molecules in strong nonresonant electric fields}

\subsection{Permanent dipole interaction}

In an electrostatic field $\varepsilon$, a polar ${ }^{1} \Sigma$ molecule with a body-fixed electric dipole moment $\mu$ is subject to a permanent-dipole potential

$$
V_{\mu}(\omega ; \theta)=-B \omega \cos \theta
$$

with $\omega=\mu \varepsilon / B$ a dimensionless parameter which measures the dipole's maximum potential energy 
in terms of the rotational constant $B$ of the molecule, ${ }^{1}$ and $\theta$ is the polar angle between the molecular axis and the direction of the field [5]-[7]. Since, in the basis of the field-free states $|J, M\rangle$, the $\cos \theta$ operator couples states with same $M$ but with $J$ 's that differ by \pm 1 , the eigenstates are hybrids of field-free rotor states for a fixed value of $M$ and a range of $J$ 's. Because $|J, M\rangle$ states of both even and odd parity contribute to such a linear superposition, the hybrid states have indefinite parity, and thus can be oriented in the space-fixed frame. The eigenproperties of the states created by the pendular potential (1) can be found by standard numerical methods. In the high-field limit, $\omega \rightarrow \infty$, these states coincide with those of a two-dimensional angular harmonic oscillator (harmonic librator) whose equidistant eigenenergies are

$$
E_{\tilde{J}, M}=B\left[-\omega+(2 \tilde{J}-|M|+1)(2 \omega)^{1 / 2}\right]
$$

The eigenstates, at any $\omega$, are labeled by $|M|$ and the nominal value $\tilde{J}$ of the angular momentum of the field-free rotor state $(\omega \rightarrow 0)$ that adiabatically correlates with the hybrid function, see Figure 1. Thus each state is characterized solely by its $\tilde{J}$ and $|M|$ and the value of $\omega$. In the harmonic librator limit, the uncertainly principle for pendular oscillations can be cast in the form $\left\langle\mathbf{J}^{2}\right\rangle\left\langle\theta^{2}\right\rangle \geq(2 \tilde{J}-|M|+1)^{2}$. This implies that, for a given state $|\tilde{J}| M|\rangle$, , achieving a narrow angular confinement requires a wide range of $J$ 's in the hybrid wavefuntion. The orientation of the molecular axis in a given state $|\tilde{J}| M|\rangle$, is characterized by the expectation value $\langle\cos \theta\rangle_{\tilde{J},|M|}$, the orientation cosine. Note that $\theta_{\tilde{J},|M|}^{0}=\arccos \langle\cos \theta\rangle_{\tilde{J},|M|}$ is the angular amplitude of the molecular axis; hence the greater the orientation cosine the smaller the angular amplitude. By the Hellmann-Feynman theorem, the orientation cosine is given by $\langle\cos \theta\rangle_{\tilde{J},|M|}=-\partial\left(E_{\tilde{J}, M} / B\right) / \partial \omega[6]$. Thus, from eq. (2), the orientation cosine in the harmonic librator limit is simply

$$
\langle\cos \theta\rangle_{\tilde{J},|M|}=1-\frac{2 \tilde{J}-|M|+1}{(2 \omega)^{1 / 2}}
$$

\subsection{Induced dipole interaction}

In an external electric field, the electronic distribution of any molecule (or atom) becomes distorted to some extent. This distortion, governed by the molecular polarizability, results in an induced dipole moment. For experimentally feasible static fields, such induced moments are very weak, typically only on the order of $10^{-2} \mathrm{D}$ (Debye). However, far stronger induced moments, well above $1 \mathrm{D}$, can now be produced by intense laser fields, using either pulsed lasers or supermirror techniques to build up a cw cavity mode. This approach is applicable regardless of whether or not the molecule is polar (or paramagnetic).

\footnotetext{
${ }^{1}$ Note that $\omega=0.0168 \mu\left[\right.$ Debye $\varepsilon[\mathrm{kV} / \mathrm{cm}] / B\left[\mathrm{~cm}^{-1}\right]$.
} 
In a nonresonant laser field, a ${ }^{1} \Sigma$ molecule is subject to an induced-dipole potential

$$
V_{\alpha}\left(\omega_{\|}, \omega_{\perp} ; \theta\right)=-B\left(\Delta \omega \cos ^{2} \theta+\omega_{\perp}\right)
$$

where $\theta$ is the angle between the molecular axis and the $\varepsilon$-field of the laser beam [8],[9]. Here $\Delta \omega \equiv$ $\omega_{\|}-\omega_{\perp}$, and the dimensionless parameters $\omega_{\|, \perp} \equiv 2 \pi \alpha_{\|, \perp} I /(B c)$, proportional to components $\alpha_{\|}$and $\alpha_{\perp}$ of the polarizability parallel and perpendicular to the molecular axis and to the laser intensity $I=\frac{c}{4 \pi} \varepsilon^{2}$, measure the maximum potential energy of the induced dipole in terms of the rotational constant, $B .^{2}$ The corresponding eigenproperties are readily evaluated by standard methods. The isotropic part of the potential, $\omega_{\perp}$, lowers all states uniformly, and the anisotropic part, governed by $\Delta \omega$, introduces a double-well corresponding to the end-for-end symmetry of the induced -dipole interaction. Since the $\cos ^{2} \theta$ operator couples states with same $M$ but with $J$ 's that differ by 0 or \pm 2 , the resulting hybrid states are superpositions of field-free rotor states of either even or odd parity, and so have a definite parity. These states can only be aligned but not oriented.

In the high-field limit, $\Delta \omega \rightarrow \infty$, the range of $\theta$ is confined near a potential minimum and the corresponding Schrödinger equation reduces to that for a two-dimensional angular harmonic oscillator (harmonic librator) with eigenenergies

$$
\begin{aligned}
E_{\tilde{J}, M} & =B\left[-\Delta \omega-\omega_{\perp}+2 \Delta \omega^{1 / 2}(\tilde{J}+1)+\frac{|M|^{2}}{2}-\frac{\tilde{J}^{2}}{2}-\tilde{J}-1\right] & & \text { for } \tilde{J}-|M| \text { even } \\
& =B\left[-\Delta \omega-\omega_{\perp}+2 \Delta \omega^{1 / 2} \tilde{J}+\frac{|M|^{2}}{2}-\frac{\tilde{J}^{2}}{2}-\frac{1}{2}\right] & & \text { for } \tilde{J}-|M| \text { odd }
\end{aligned}
$$

The states are, again, labeled by the good quantum number $|M|$ and the nominal value $\tilde{J}$ of the angular momentum of the field-free rotor state $(\omega \rightarrow 0)$ that adiabatically correlates with the hybrid function, see Fig. 1. The uncertainty principle for the harmonic pendular oscillations can be cast in a form similar to that for the permanent-dipole case.

The spatial anisotropy of the molecular axis distribution is characterized by the expectation value $\left\langle\cos ^{2} \theta\right\rangle_{\tilde{J}, M}=-\partial\left(E_{\tilde{J}, M} / B\right) / \partial \Delta \omega$, the alignment cosine. The angular amplitude of the molecular axis is then $\theta^{0}=\arccos \left[\left\langle\cos ^{2} \theta\right\rangle\right]^{1 / 2}$. In the harmonic librator limit, we obtain from eq. (5) the alignment cosine

$$
\begin{aligned}
\left\langle\cos ^{2} \theta\right\rangle_{\tilde{J}, M} & =1-\frac{\tilde{J}+1}{\Delta \omega^{1 / 2}} & & \text { for } \tilde{J}-|M| \text { even } \\
& =1-\frac{\tilde{J}}{\Delta \omega^{1 / 2}} & & \text { for } \tilde{J}-|M| \text { odd }
\end{aligned}
$$

The nonresonant radiation can also be delivered as a pulse of intensity $I(t)=\left\langle\varepsilon^{2}(t)\right\rangle c /(4 \pi)$ $=I_{0} g(t / \tau)$ where $I_{0}$ denotes the peak intensity and $g(t / \tau)$ the pulse time profile, with $\tau$ the pulse

\footnotetext{
${ }^{2}$ Note that $\Delta \omega=10^{-11} \Delta \alpha\left[\AA^{3}\right] I\left[\mathrm{~W} / \mathrm{cm}^{2}\right] / B\left[\mathrm{~cm}^{-1}\right] ; I\left[\mathrm{~W} / \mathrm{cm}^{2}\right]=2.654 \times 10^{3} \varepsilon^{2}\left[\mathrm{kV}^{2} / \mathrm{cm}^{2}\right]$.
} 
duration. Here we have assumed that the oscillation frequency is far removed from any molecular resonance and much higher than the rotational periods. As a result, $\Delta \omega=\Delta \omega(t)$ and so $V_{\alpha}=V_{\alpha}(t)$ becomes a function of time. The analysis [10] of the time dependence shows that in the short-pulse limit $(\tau \ll \pi \hbar / B)$, the interaction is non-adiabatic and the pendular states recur after the pulse had passed, making it possible and feasible to obtain molecular alignment under field-free conditions. In the long-pulse limit $(\tau \approx \pi \hbar / B)$, the interaction is adiabatic and pendular states faithfully follow the field as if it were static at any instant. It is this latter case that we'll limit our considerations to.

\section{Net polarization of a molecular beam}

When a molecular beam enters into the range of an electrostatic field, the populations of the field-free rotational states are adiabatically transferred to the hybrid states created by the field. A similar adiabatic population transfer takes place when the beam molecules are irradiated by a laser pulse whose duration $\tau$ exceeds $\pi \hbar / B$. In either case, each initial rotational state $J$ is being

redistributed $(J \rightarrow \tilde{J})$ into $(2 \tilde{J}+1)$ states within the field with a statistical weight given by the Boltzmann factor

$$
w_{\tilde{J}}=\exp [-\tilde{J}(\tilde{J}+1) / \Upsilon] Q^{-1}
$$

which depends on the reduced rotational temperature

$$
\Upsilon \equiv \frac{k T}{B}
$$

and the rotational partition function,

$$
Q \equiv \sum_{\tilde{J}=0}^{\infty}(2 \tilde{J}+1) \exp [-\tilde{J}(\tilde{J}+1) / \Upsilon]=Q(\Upsilon)
$$

Here $T$ is the rotational temperature of the beam molecules and $B$ is their rotational constant.

We note that the distinguishing feature of our treatment here is that the molecules maintain their free-rotor Boltzmann factors while in the field. In other words, the eigenenergies the molecules acquire in the field do not enter their Boltzmann factors. This is exactly the situation encountered in the case of an adiabatic population transfer of beam molecules from a field-free to a high-field region in the absence of a relaxation mechanism (such as collisions): in such a case the molecules will not establish any new equilibrium populations in the field.

Figure 1 shows a correlation diagram between the free-rotor states $\tilde{J}$ and the harmonic librator limits for the permanent dipole interaction and the induced-dipole interaction, see caption. It is according to this scheme that the populations of the free-rotor states are distributed among the hybrid $|\tilde{J}| M|\rangle$, states. Note the characteristic differences in the level structure of the permanent 
and induced dipole cases, such as the opposite ordering of the $|M|$ states for a given $\tilde{J}$ and the $(2 \omega)^{1 / 2}$ and $2 \Delta \omega^{1 / 2}$ spacings of the respective harmonic librator levels.

\subsection{Net orientation of permanent dipoles}

The net orientation (i.e., the ensemble average of the orientation cosine) is given by

$$
\langle\langle\cos \theta\rangle\rangle=\sum_{\tilde{J}=0}^{\infty} w_{\tilde{J}} \sum_{M=-\tilde{J}}^{M=+\tilde{J}}\langle\cos \theta\rangle_{\tilde{J}, M}
$$

We'll now make use of the analytic expression for the orientation cosine in the harmonic

librator limit, eq. (3), and substitute it into eq. (10). As a result, the sum over $M$ in eq. (10) becomes explicit and, moreover, can be carried out in closed form:

$$
\begin{aligned}
\sum_{M=-\tilde{J}}^{M=+\tilde{J}}\langle\cos \theta\rangle_{\tilde{J}, M} & =2 \tilde{J}+1-\frac{(2 \tilde{J}+1)(2 \tilde{J}+1)-\tilde{J}(\tilde{J}+1)}{(2 \omega)^{1 / 2}} \\
& =2 \tilde{J}+1-\frac{3 \tilde{J}^{2}+3 \tilde{J}+1}{(2 \omega)^{1 / 2}}
\end{aligned}
$$

In order to complete our closed-form evaluation of the net orientation cosine, we'll replace the summation over $\tilde{J}$ by integration over a continuous variable $x, \tilde{J} \rightarrow x$. As a result,

$$
\begin{aligned}
\langle\langle\cos \theta\rangle\rangle & =Q^{-1} \sum_{\tilde{J}=0}^{\infty} \exp [-\tilde{J}(\tilde{J}+1) / \Upsilon]\left[2 \tilde{J}+1-\frac{3 \tilde{J}^{2}+3 \tilde{J}+1}{(2 \omega)^{1 / 2}}\right] \\
& \rightarrow Q^{-1} \int_{0}^{\infty} \exp [-x(x+1) / \Upsilon]\left[2 x+1-\frac{3 x^{2}+3 x+1}{(2 \omega)^{1 / 2}}\right] d x \\
& =1-\frac{1}{(2 \omega)^{1 / 2}}\left\{\frac{3}{4}+\frac{1}{8}\left(\frac{\pi}{\Upsilon}\right)^{1 / 2}(1+6 \Upsilon) \exp \left[\frac{1}{4 \Upsilon}\right]\left[1-\operatorname{erf}\left(\frac{1}{2 \Upsilon^{1 / 2}}\right)\right]\right\}
\end{aligned}
$$

where we made use of

$$
\begin{aligned}
Q(\Upsilon) & =\sum_{\tilde{J}=0}^{\infty}(2 \tilde{J}+1) \exp [-\tilde{J}(\tilde{J}+1) / \Upsilon] \\
& \rightarrow \int_{0}^{\infty}(2 x+1) \exp [-x(x+1) / \Upsilon] d x=\Upsilon
\end{aligned}
$$

i.e., that at high reduced rotational temperatures the rotational partition function coincides with the reduced rotational temperature.

\subsection{Net alignment of induced dipoles}

The net alignment (i.e., the ensemble average of the alignment cosine) is given by

$$
\left\langle\left\langle\cos ^{2} \theta\right\rangle\right\rangle=\sum_{\tilde{J}=0}^{\infty} w_{\tilde{J}} \sum_{M=-\tilde{J}}^{M=+\tilde{J}}\left\langle\cos ^{2} \theta\right\rangle_{\tilde{J}, M}
$$


Substituting for the alignment cosine from eq. (6) allows the sum over $M$ to be, again, evaluated in closed form:

$$
\begin{aligned}
\sum_{M=-\tilde{J}}^{M=+\tilde{J}}\left\langle\cos ^{2} \theta\right\rangle_{\tilde{J}, M} & =\tilde{J}-\frac{\tilde{J} \tilde{J}}{\Delta \omega^{1 / 2}}+\tilde{J}+1-\frac{(\tilde{J}+1)(\tilde{J}+1)}{\Delta \omega^{1 / 2}} \\
& =2 \tilde{J}+1-\frac{2 \tilde{J}^{2}+2 \tilde{J}+1}{\Delta \omega^{1 / 2}}
\end{aligned}
$$

where the first two terms on the right-hand side of the first line correspond to states with $\tilde{J}-|M|$ odd and the last two terms to $\tilde{J}-|M|$ even. Now, again, we'll replace the summation over $\tilde{J}$ by integration over a continuous variable $x, \tilde{J} \rightarrow x$, and obtain as a result,

$$
\begin{aligned}
\left\langle\left\langle\cos ^{2} \theta\right\rangle\right\rangle & =\sum_{\tilde{J}=0}^{\infty} w_{\tilde{J}}\left[2 \tilde{J}+1-\frac{2 \tilde{J}^{2}+2 \tilde{J}+1}{\Delta \omega^{1 / 2}}\right] \\
& \rightarrow Q^{-1} \int_{0}^{\infty} \exp [-x(x+1) / \Upsilon]\left[2 x+1-\frac{2 x^{2}+2 x+1}{\Delta \omega^{1 / 2}}\right] d x \\
& =1-\frac{1}{2 \Delta \omega^{1 / 2}}-\left(\frac{\pi}{16 \Upsilon \Delta \omega}\right)^{1 / 2}(1+2 \Upsilon) \exp \left[\frac{1}{4 \Upsilon}\right]\left[1-\operatorname{erf}\left(\frac{1}{2 \Upsilon^{1 / 2}}\right)\right]
\end{aligned}
$$

\section{Validity range of the net polarization formulae}

The validity of the analytic expressions (12) and (16) for the net orientation and alignment of molecules in a beam can be best assessed by a comparison with the corresponding exact numerical calculation. Such a comparison is made in Figure 2 for the net orientation and in Figure 3 for the net alignment, over a wide range of reduced temperatures $\Upsilon$ and interaction parameters $\omega$ or $\Delta \omega$. The numerical calculations were carried out by diagonalizing the corresponding Hamiltonian matrix, extracting the eigenvectors, computing the orientation/alignment cosines for each individual state, and, finally, carrying out the summations over the $\tilde{J}$ and $M$ states according to eqs. (10) or (14). Such a computation is quite onerous: for instance, at $\Upsilon=100$ and $\omega=100$, about a hundred $100 \times 100$ matrices need to be diagonalized in order to obtain a result that converges within $1 \%$.

From Figs. 2 and 3 we see that the agreement of our approximation (shown by the grey curves) with the exact calculations (shown by black curves) becomes excellent at about $\omega / \Upsilon \gtrsim 3$ for the net orientation and at about $\Delta \omega / \Upsilon \gtrsim 5$ for the net alignment.

One may wonder about the reasons for the wide applicability of formulae (12) and (16). Obviously, at high-enough values of $\omega$ or $\Delta \omega$, all states populated at a given $\Upsilon$ will become harmonic librator states. This is indeed the regime where our formulae are exact. What may come as a 
surprise is how low a value of $\omega$ or $\Delta \omega$ at a given $\Upsilon$ is required to reach the harmonic limit. This is well illustrated already for the ground state (i.e., $\Upsilon=0$ ): apart from the singularity at $\omega, \Delta \omega \rightarrow 0$, the ground-state harmonic librator orientation/alignment cosine comes close to an exact value at $\omega, \Delta \omega \gtrsim 1$, indicating that from the value $\omega, \Delta \omega \approx 1$ on, the $|\tilde{J}=0| M,|=0\rangle$ state is securely bound in the parabolic neighborhood of either of the pendular potentials (1) or (4). Similarly for higher states populated at higher $\Upsilon$. The most probable value of $\tilde{J}$ at a given $\Upsilon$ is

$$
\tilde{J}^{*} \approx\left(\frac{\Upsilon}{2}\right)^{1 / 2}
$$

which reveals that states of up to about $3 \tilde{J}^{*}$ need to be hybridized in order to attain the harmonic limit. And they do ... Indeed, evaluating the net polarization in the harmonic librator limit as we did gives us a better sense for the effect of the pendular potentials and the onset of the harmonic limit.

The somewhat different validity ranges of formulae (12) an (16) can be accounted for by noticing the difference in the spacings of the harmonic librator levels for the permanent- and induced-dipole cases, cf. eqs. (2) and (5): this is greater for the induced dipole interaction than for the permanent dipole by a factor of $2^{1 / 2}$ at $\omega=\Delta \omega$, cf. Fig. 1. Hence a correspondingly greater value of $\Delta \omega$ needs to be applied in order to reach the harmonic limit for the induced-dipole case (namely, $\Delta \omega / \Upsilon \gtrsim 5)$ than for the permanent-dipole interaction $(\omega / \Upsilon \gtrsim 3)$.

We note that a series expansion for $\Upsilon \rightarrow \infty$ of formula (12) works as well as the formula itself at $\Upsilon \gtrsim 10$. The expansion (up to 1 st order in $\Upsilon$ ) takes the form

$$
\langle\langle\cos \theta\rangle\rangle \rightarrow 1-\frac{1}{(8 \omega \Upsilon)^{1 / 2}}\left[\frac{4}{\Upsilon^{1 / 2}}+\pi^{1 / 2}(12 \Upsilon-5)\right] \quad \text { at } \Upsilon \text { large }
$$

Even better, for the net alignment, a series expansion for $\Upsilon \rightarrow \infty$ of formula (16) works as well as the formula itself at all $\Upsilon$. The expansion (up to 1st order in $\Upsilon$ ) takes the form

$$
\left\langle\left\langle\cos ^{2} \theta\right\rangle\right\rangle \rightarrow 1-\frac{1}{2 \Delta \omega^{1 / 2}}\left[1+\left(\left(\frac{\pi}{16 \Upsilon}\right)^{1 / 2}(3+4 \Upsilon)-\frac{2}{3 \Upsilon}-1\right)\right]
$$

Therefore, formulae (19) and (16) can be used interchangeably.

In deriving formulae (12) and (16), we made use of the Euler-Maclaurin formula to replace sums by integrals [11]. Interestingly, the remainder in the Euler-Maclaurin formula is smaller for the net-polarization formulae than it is, e.g., for the rotational partition function, eq. (13). In fact, $\left|\sum-\int\right| /\left(\sum+\int\right) \lesssim 0.15$ within the validity range of formulae (12) and (16). Moreover, the deviation of the integral from the sum is such that the resultant approximation (grey curves in Figs. 2 and 3) comes closer to the exact result (black curves) than it would have if it were based on the numerically evaluated sums. 
In our previous work [12] we provided nomograms that summarize the attainable values of the interaction parameters $\omega$ and $\Delta \omega$ as a function of field strength or laser intensity for a variety of molecules. Since field strengths $\varepsilon \approx 100 \mathrm{kV} / \mathrm{cm}$ can be attained in electrostatic fields, $\omega$ values on the order of 10, sometimes even 1000, can be obtained. For instance, for the trimer $(\mathrm{HCN})_{3}$, whose rotational constant is small and dipole moment large, a value of $\omega \approx 600$ has been achieved [13],[14]. Available cw lasers combined with build-up cavities give promise of delivering up to $10^{11}$ $\mathrm{W} / \mathrm{cm}^{2}$ at a narrow beam waist. This corresponds to a field strength of about $10^{7} \mathrm{~V} / \mathrm{cm}$, sufficient to induce a dipole moment on the order of $1 \mathrm{D}$ in a typical small molecule such as $\mathrm{Cl}_{2}$. Pulsed lasers can deliver far higher intensities, although spectroscopic or trapping experiments will usually not want to exceed about $10^{12} \mathrm{~W} / \mathrm{cm}^{2}$ in order to avoid ionizing the molecules. The corresponding values of the $\Delta \omega$ parameter then fall between 100 and 1000 for typical small molecules.

Table I lists, for a selection of rotational temperatures and rotational constants, the values of the reduced temperature, along with $\tilde{J}^{*}$ and the minimum values $\omega^{\diamond}$ and $\Delta \omega^{\diamond}$ of the interaction parameters $\omega$ and $\Delta \omega$ for which formulae (12) and (16) still apply.

\section{Conclusions}

We have developed a simple analytic approximation for evaluating the net orientation and alignment of molecules in a beam. The validity of the resultant formulae at $\omega / \Upsilon \gtrsim 3$ (net orientation, eq. (12)) and $\Delta \omega / \Upsilon \gtrsim 5$ (net alignment, eq. (16 )) was established by a comparison with an exact numerical calculation. The formulae should find wide application in assessing the overall polarization achieved in molecular beams interacting with external fields as well as in other situations where molecules maintain their field-free populations while interacting with a field. These include molecules embedded in small He nano-droplets, subjected to an external field, as revealed by the experiments of Nauta and Miller [15].

Acknowledgment. I'm grateful to Gerard Meijer (Berlin), Wieland Schöllkopf (Berlin), and Roger Miller (Chapel Hill) for their helpful comments. I dedicate this paper to the memory of Roger Miller. 


\section{References}

[1] P. Langevin, J. de phys. 4, 678 (1905).

[2] P. Debye, Polar molecules (New York: Chemical Catalogue, reprinted by Dover, 1929).

[3] J.H. Van Vleck, The theory of electric and magnetic susceptibilities (Oxford University Press, 1932).

[4] B. Friedrich and D. Herschbach, Int. Rev. Phys. Chem. 15, 325 (1996) and work cited therein.

[5] H.J. Loesch, Annu. Rev. Phys. Chem. 46, 555 (1995) and work cited therein.

[6] B. Friedrich, A. Slenczka, and D. Herschbach, Can. J. Phys. 72, 897 (1994).

[7] A. Slenczka, Chem. Eur. J. 5, 1136 (1997) and work cited therein.

[8] B. Friedrich and D. Herschbach, Phys. Rev. Lett. 74, 4623 (1995).

[9] B. Friedrich and D. Herschbach, J. Phys. Chem. 99, 15686 (1995).

[10] J. Ortigoso, M. Rodriguez, M. Gupta, and B. Friedrich, J. Chem. Phys. 110, 3870 (1999).

[11] H.P. Boas and C. Stutz, Amer. J. Phys. 39, 745 (1971).

[12] B. Friedrich, D. Pullman, and D. Herschbach, J. Phys. Chem. 95, 8118 (1991).

[13] P.A. Block, E.J. Bohac, and R.E. Miller, Phys. Rev. Lett. 68, 1303 (1992).

[14] J.-M. Rost, J.C. Griffin, B. Friedrich, and D. Herschbach, Phys. Rev. Lett. 68, 1299 (1992).

[15] K. Nauta and R. E. Miller, J. Chem. Phys. 117, 4846 (2002). 


\section{Figure Captions}

Figure 1: Correlation diagram between the free-rotor states $\tilde{J}$ (levels at the center) and the harmonic librator limits for the permanent-dipole interaction (levels on the left) and the induced-dipole interaction (levels on the right). The equidistant levels in the harmonic librator limit for either the permanent- or induced-dipole case are labeled by the librator quantum number $N$ and the projection $|M|$ of the angular on the field vector. For the permanent dipole interaction, $N=2 \tilde{J}-|M|$; for the induced dipole interaction, $N=\tilde{J}$ for $\tilde{J}-|M|$ even and $N=\tilde{J}-1$ for $\tilde{J}-|M|$ odd.

Figure 2: Dependence of the net orientation $\langle\langle\cos \theta\rangle\rangle$ (i.e., ensemble-averaged expectation value of the cosine of the polar angle $\theta$ between the molecular axis and electric-field vector) as a function of the permanent-dipole interaction parameter $\omega$ (proportional to the strength of the electric field) at different values of the reduced rotational temperature $\Upsilon$. The exact numerical calculations are shown by black curves and the model, formula (12), by the grey curves. Once overlap is reached, only the model results are shown.

Figure 3: Dependence of the net alignment $\left\langle\left\langle\cos ^{2} \theta\right\rangle\right\rangle$ (i.e., ensemble-averaged expectation value of the square of the cosine of the polar angle $\theta$ between the molecular axis and electric-field vector) as a function of the induced-dipole interaction parameter $\Delta \omega$ (proportional to the square of the strength of the electric field or linearly proportional to laser intensity) at different values of the reduced rotational temperature $\Upsilon$. The exact numerical calculations are shown by black curves and the model, formula (16), by the grey curves. Once overlap is reached, only the model results are shown. 
Table I: A sampling of rotational temperatures, $T$, and reduced rotational temperatures, $\Upsilon{ }^{a}$ that obtain for a selection of rotational constants, $B$, along with the most probable rotational state $\tilde{J}^{*}$, and the minimum values $\omega^{\diamond}$ and $\Delta \omega^{\diamond}$ of the dimensionless interaction parameters for which formulae (12) and (16) are valid. See text.

\begin{tabular}{c|c|c|c|c|c}
\hline$T[\mathrm{~K}]$ & $B\left[\mathrm{~cm}^{-1}\right]$ & $\Upsilon$ & $\tilde{J}^{*}$ & $\omega^{\diamond}$ & $\Delta \omega^{\diamond}$ \\
\hline \hline 10 & 1 & 7 & 2 & 21 & 35 \\
\hline 10 & 0.1 & 70 & 6 & 210 & 350 \\
\hline 10 & 0.01 & 700 & 19 & 2100 & 3500 \\
\hline 1 & 1 & 0.7 & 0 & 2 & 3.5 \\
\hline 1 & 0.1 & 7 & 2 & 21 & 35 \\
\hline 1 & 0.01 & 70 & 6 & 210 & 350 \\
\hline 0.38 & 1 & 0.3 & 0 & 1 & 1 \\
\hline 0.38 & 0.1 & 2.7 & 1 & 8 & 14 \\
\hline 0.38 & 0.01 & 27 & 4 & 81 & 140 \\
\hline \hline
\end{tabular}

${ }^{a}$ The reduced rotational temperature $\Upsilon=0.69 T[\mathrm{~K}] / B\left[\mathrm{~cm}^{-1}\right]$. 
Permanent

dipole

$N_{|M|}$

Free

rotor

$\tilde{J}$

$\tilde{J},|M|$

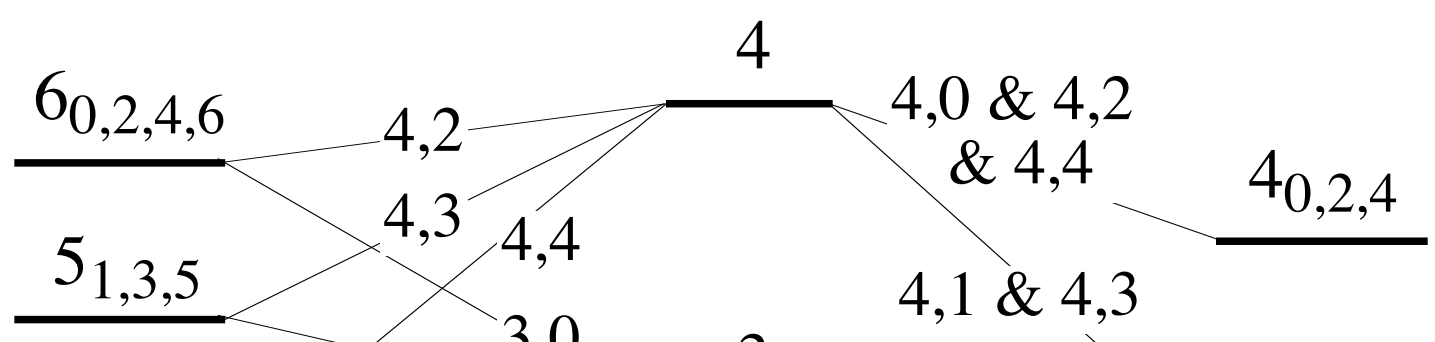

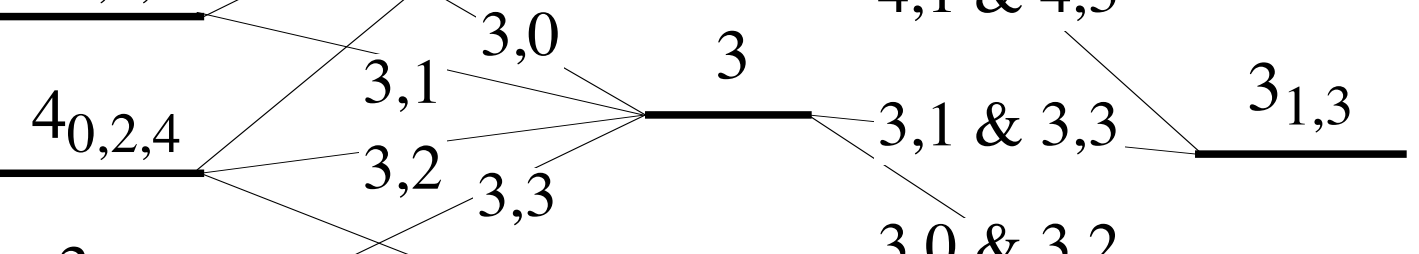

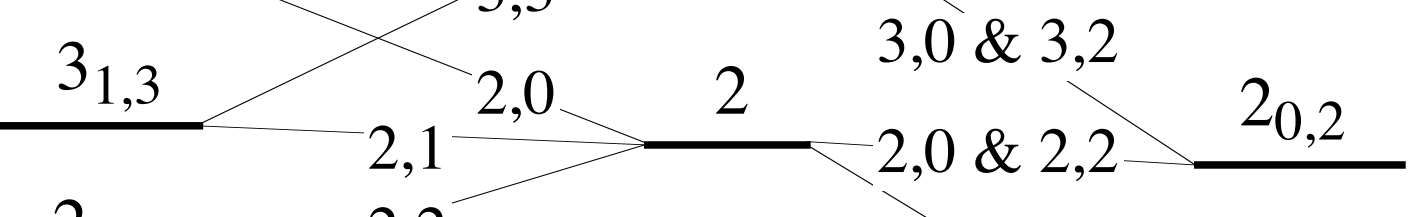

$\frac{2_{0,2}}{1_{1}}-1,2$
$\frac{(2 \omega)^{1 / 2}}{0_{0}}-0,0$

Figure 1 


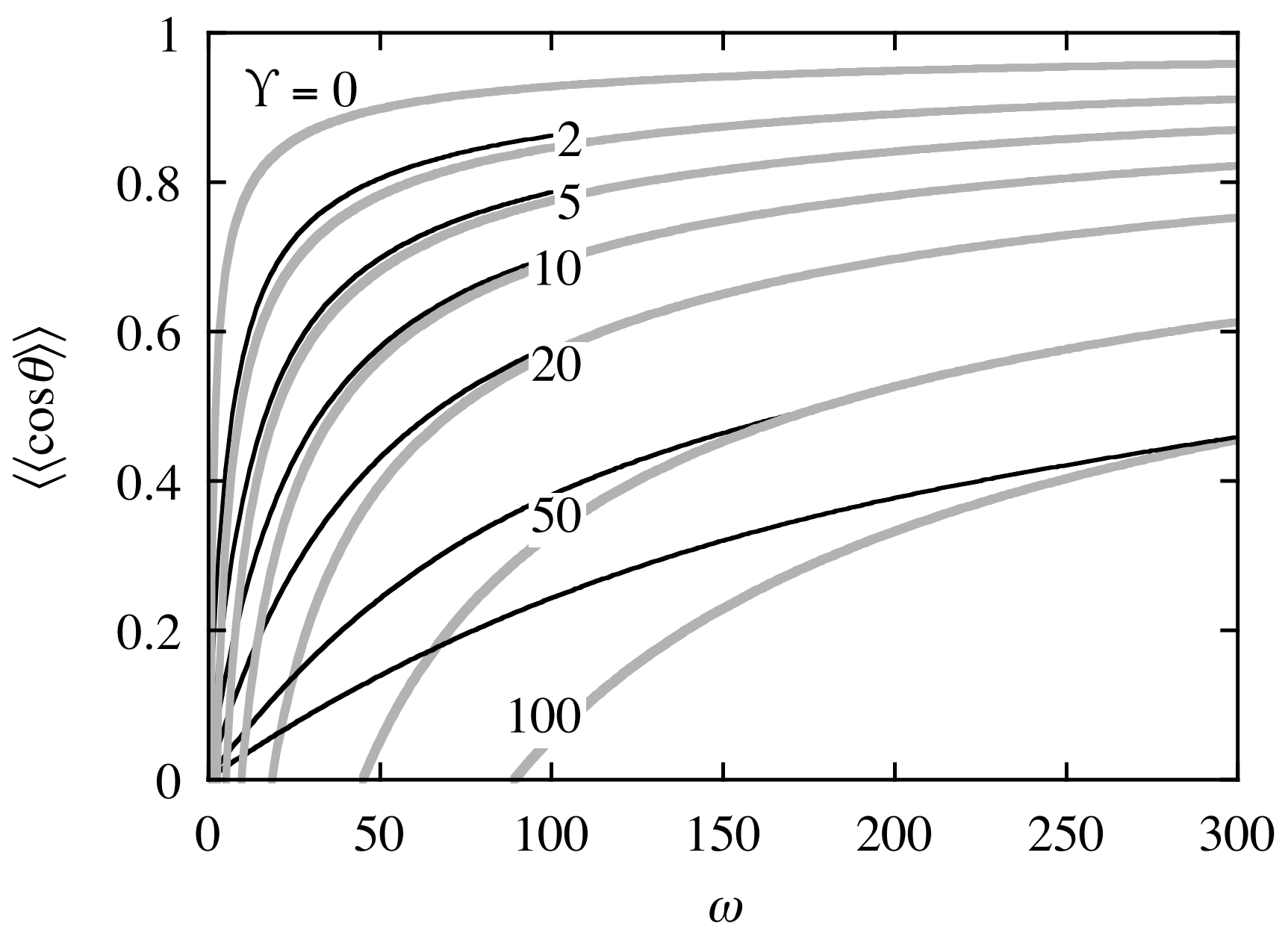

Figure 2 


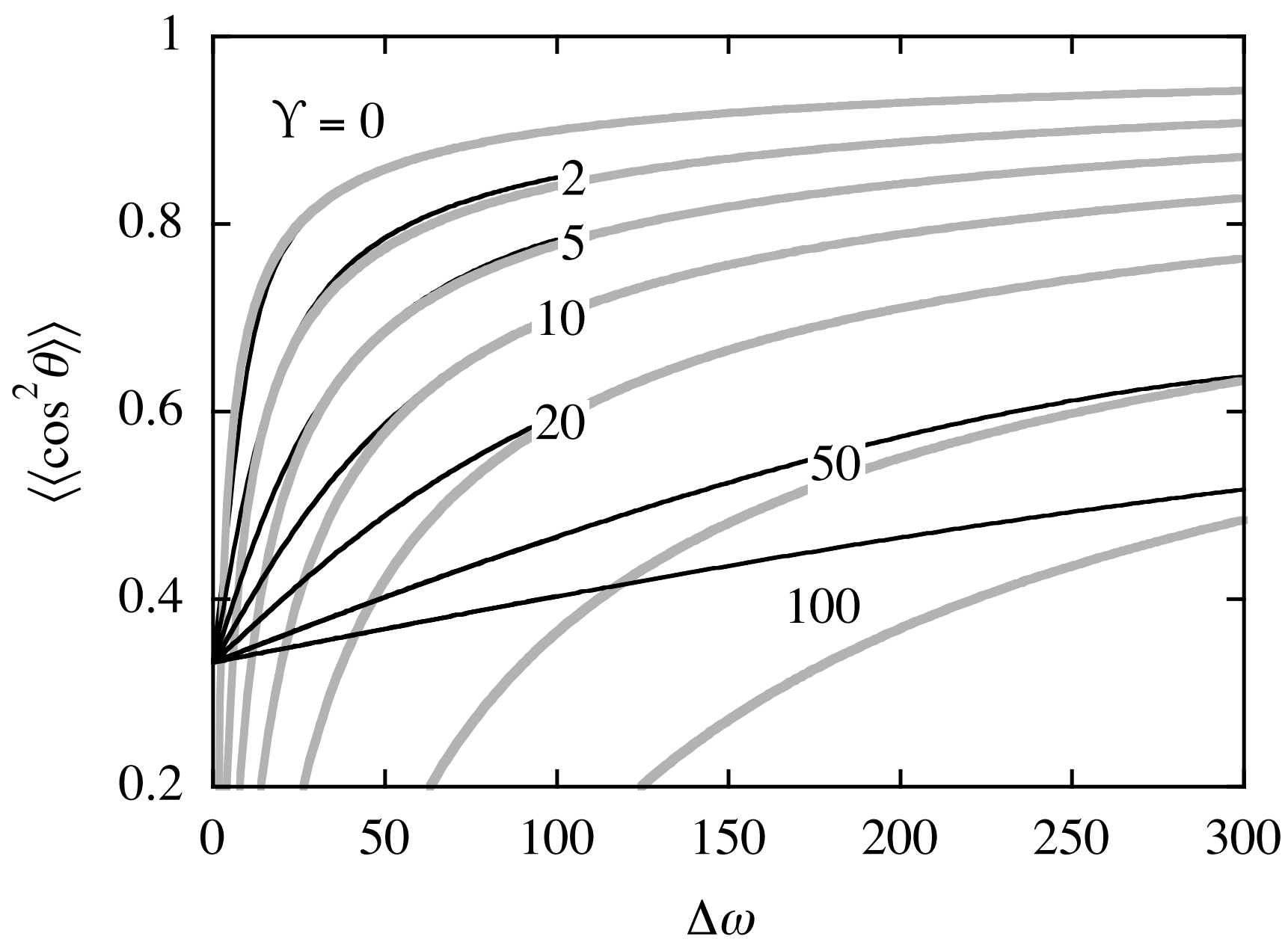

Figure 3 